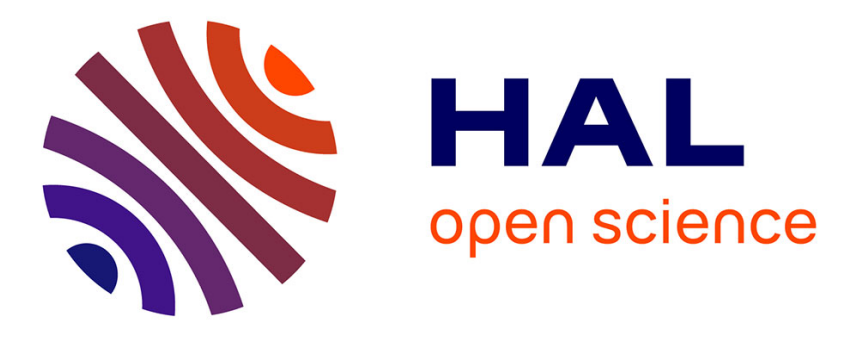

\title{
3D underwater reconstruction of archeology object with a mono camera camcorder: The case study at Gourneyras lake
}

Yadpiroon Onmek, Jean Triboulet, Sébastien Druon, Arnaud Meline, Silvain Louis, Bruno Jouvencel

\section{To cite this version:}

Yadpiroon Onmek, Jean Triboulet, Sébastien Druon, Arnaud Meline, Silvain Louis, et al.. 3D underwater reconstruction of archeology object with a mono camera camcorder: The case study at Gourneyras lake. OCEANS, Jun 2017, Aberdeen, United Kingdom. pp.1-6, 10.1109/OCEANSE.2017.8084978 . lirmm-02018544

\section{HAL Id: lirmm-02018544 https://hal-lirmm.ccsd.cnrs.fr/lirmm-02018544}

Submitted on 22 May 2019

HAL is a multi-disciplinary open access archive for the deposit and dissemination of scientific research documents, whether they are published or not. The documents may come from teaching and research institutions in France or abroad, or from public or private research centers.
L'archive ouverte pluridisciplinaire HAL, est destinée au dépôt et à la diffusion de documents scientifiques de niveau recherche, publiés ou non, émanant des établissements d'enseignement et de recherche français ou étrangers, des laboratoires publics ou privés. 


\title{
3D Underwater Reconstruction of Archeology object with a monocamera camcorder: Case study at Gourneyras lake
}

\author{
Y. Onmek ${ }^{*}$, J. Triboulet ${ }^{\dagger}$, S. Druon ${ }^{\ddagger}$, A. Meline ${ }^{\S}$, B. Jouvencel ${ }^{\mathbb{T}}$ \\ LIRMM Laboratory, University of Montpellier - CNRS UMR 5506, \\ 161 rue Ada, 34090 Montpellier Cedex 5, France \\ Email: *Omnec@lirmm.fr, ${ }^{\dagger}$ Triboulet@lirmm.fr, ${ }^{\ddagger}$ Druon@lirmm.fr, ${ }^{\S}$ Meline@ lirmm.fr, IIJouvencel@ lirmm.fr
}

\begin{abstract}
This work presents the 3D reconstruction of the archeology objects in underwater environment. The videos and images are obtained from a calibrated camera system. The features of interest between image pairs are selected by the FAST detector and FLANN descriptor. Subsequently, the RANSAC method is applied to reject outlier points. The putative inliers are matched by triangulation to produce sparse point clouds in 3D space, using a pinhole camera model and Euclidean distance estimation. The 3D model textrue is achieved through a commond 3D Delaunay triangulation procedure. Moreover, evaluation of the quality of the underwater $3 \mathrm{D}$ reconstruction model to find its accuracy is done by measurement, comparing it with a 3D industrial scan model as well as a real object.
\end{abstract}

Keywords: 3D reconstruction, Underwater Archeology, Monocamera, Scanner

\section{INTRODUCTION}

In recent years, 3D imaging sensors and $3 \mathrm{D}$ reconstruction from single camera-vision systems have increased in popularity in the computer vision community, computer graphics, and photogrammetry. The essential 3D modeling applications are robot navigation, visual inspection, virtual reality, and so on. In the context of archeology, several researchers have been developing novel approaches to produce 3D models of objects as well as scenes.

Numerous methods exist, but they are not applicable to all objects and environments. Indeed, they depend on knowledge of the system as well as the environments. Especially in underwater environments, there are several limitations when working with underwater images. It is not easy to access and recover $3 \mathrm{D}$ information because of the possibility of encountering poor experimental conditions. More importantly, in underwater environment we must deal with various conditions for instant light conditions, loss of color and contrast in significant depth, the effects of several noises, unclear water, as well as poor data acquisition control. The main purpose of this paper is the evaluation of $3 \mathrm{D}$ reconstruction methods for archeological objects (a female bust) in underwater environments.

The underwater video and images sequence for this work were acquired by divers at the Gourneyras lake Fig 1 located in the Herault department in southern France.First using a small single digital camera to take a video of a calibration chessboard to calibrate the parameters of the camera. The initial image pairs are captured and extracted from a calibrated single camera camcorder system. The features of interest are selected and matched across image pairs. An additional outlier removal step is performed based on the RANSAC method.
Triangulation of the inlier features from the $2 \mathrm{D}$ images space into a sparse 3D points cloud is done by using a pinhole camera model and Euclidean distance estimation. Then, the texture and rendering of the $3 \mathrm{D}$ stereo model is procedure. Furthermore, evaluation of the $3 \mathrm{D}$ reconstruction model quality is done by a measurement comparison with a $3 \mathrm{D}$ industrial scan model as well as the real object. The 3D reconstruction pipeline is presented in Fig 2.

This paper is organized as follows: In section II a brief overview of 3D reconstruction problems is presented. Section III describes the method applied for 3D reconstruction in the case of an underwater environment: the initial instrinsic camera parameters are provided by a calibration procedure, the detector and discription methods for correspondence problems as well as the reconstruction techniques are described. The evaluations of the system and results are described with noisesimulating underwater conditions in section IV. In the last section, discussion, conclusions as well as future works are presented.

\section{RELATED WORKS}

This section briefly discusses some earlier works related to the detection of features of interest, matching point-sets as well as the reconstruction of 3D models. Comparative performances of methods have been published, assessing the detection performance features and image matching algorithms. The system of [13] compared several well-known feature detectors and descriptors. To compare each combination performance objectively, the effects of JPEG compression, zoom and rotation, blur, viewpoint as well as illumination variations have been investigated in terms of precision and recall values. Similarly, works from [16], proposed to investigate the performance of the SIFT and HARRIS methods. They simulated a noise filter in surface images to compare the percentage of inliers. More recent systems built on this same approach [1] considered methods to improve color quality and contrast of underwater images that do not need a prior knowledge of the scene. Finally, SIFT and SURF descriptors were used to compare the computation time.

The procedure of some researchers was to create a digital model and physical replicas [2] using undetermined images to estimate 3D urban models. They give no information about the devices that took the pictures, but their methods engage a prior knowledge of the scene. In the same way,[22] proposed a method for $3 \mathrm{D}$ object reconstruction to elaborate the 3D 


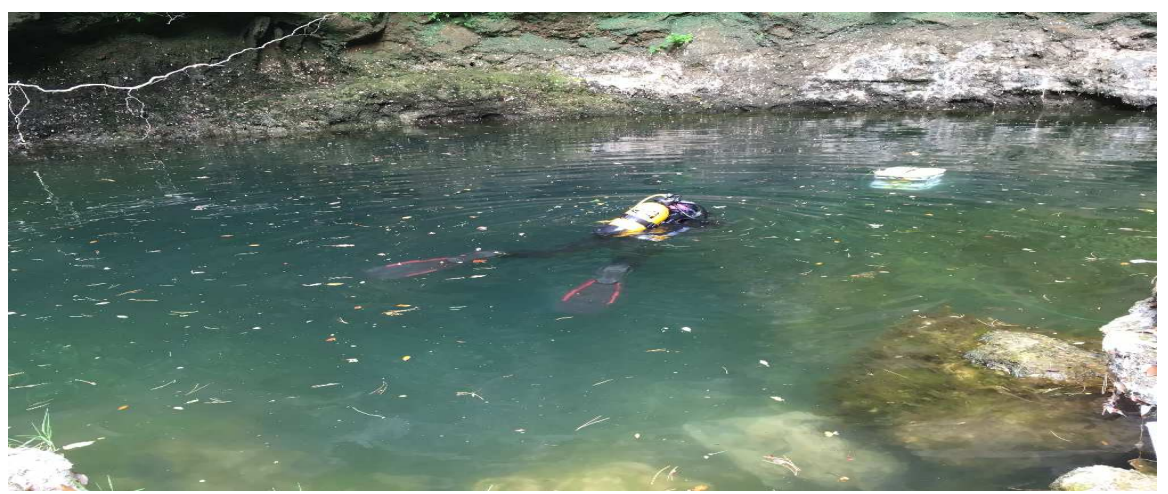

Fig. 1: The Gourneyras lake, Herault department in south of France.

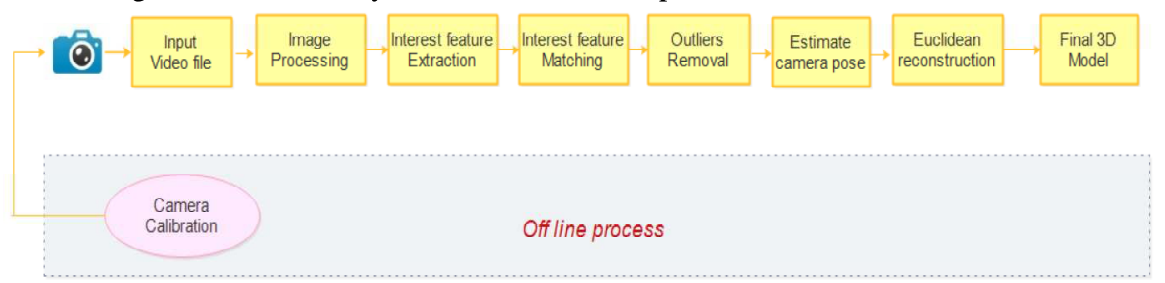

Fig. 2: The 3D underwater reconstruction system overview.

model from pictures in an automated way. Concerning robust photometric implementation, a bundle adjustment is used to create a standard package. Another essential point in [8] presented a multi-view stereo algorithm capable of computing high quality reconstruction of a range of scenes from large, shared, multiuser picture collections available on the Internet. This capability opens the possibility of computing accurate geometric models of several sites such as cities and landscapes.

Focusing on underwater reconstruction, [15] stated that to carry out the $3 \mathrm{D}$ reconstruction of natural underwater scenes from images obtained from calibrated single cameras, underwater constraints in the camera distortion model must be taken into account. Consequently, [12] combined a 3 DOF inertial sensor and a calibrated stereo rig to estimate the trajectory and produced a 3D dense map. [4] used a stereo camera to capture images and reconstruct indoor environments for robot navigation.

\section{METHODS}

This section presents all of the methods and materials used in this paper.

\section{A. Camera calibration}

In $3 \mathrm{D}$ computer vision fields, camera calibration is a mandatory step, and it is an important task for Euclidean reconstruction [25]. The process of camera calibration is to impose the characteristics of the transformation between an object in $3 \mathrm{D}$ space and the 2D image observed by a camera. The transformation includes the characteristics of the camera, such as intrinsic parameters including focal length, the principal points of the camera as well as distortion, and extrinsic parameters to present the orientation and camera locations, such as a rotation matrix and translation vector.
1) Intrinsic parameter: The camera model used is the classical pinhole camera model.The intrinsic matrix transforms 3D camera cooordinates to $2 \mathrm{D}$ homogeneous image coordinates. This perspective projection is modeled by the ideal pinhole camera. The intrinsic matrix is parameterized by [11].

$$
K=\left[\begin{array}{ccc}
f x & s & C x \\
0 & f y & C y \\
0 & 0 & 1
\end{array}\right]
$$

where $f x$ and $f y$ are focal lengths, $s$ is the the skew, which is 0 in our work, and the principal point $(c x, c y)$. The extrinsic calibration parameters consist of the $3 \times 3$ rotation matrix $R$ and the $3 x 1$ translation vector $t$ which describe the pose of the camera movement are presented in section 3.5.

\section{B. Image Processing}

In case of underwater images somethimes need to improve or filter images before $3 \mathrm{D}$ reconstruction processing. Because, it have noise in the images that effect from light, deep or sand, dirty water.

1) Gaussian filter: In image processing, a Gaussian blur [19] (also known as Gaussian smoothing) is the result of blurring an image by a Gaussian function. It is a widely used effect in graphics software, typically to reduce image noise and reduce detail. The visual effect of this blurring technique is a smooth blur resembling that of viewing the image through a translucent screen, distinctly different from the bokeh effect produced by an out-of-focus lens or the shadow of an object under usual illumination. Gaussian smoothing is also used as a pre-processing stage in computer vision algorithms in order to enhance image structures at different scalessee scale space representation and scale space implementation. 
2) Median filter: The median filter is a nonlinear digital filtering technique [19], it is used to remove "salt and pepper" noise. The template size slider defines how much filtering takes place. Median filtering is a nonlinear method used to remove noise from images.It is widely used as it is very effective at removing noise while preserving edges.

\section{Detection and Extraction}

The next step is to find the correspondence between the first and second images. In other words, when a scene or an object must be reconstructed in $3 \mathrm{D}$, detection and matching points in the images are the most crucial factors for model accuracy. The 3D model will be of low quality or even completely wrong if the feature extraction and matching steps introduce errors. We investigated several feature detectors and many of them are very good, such as the basic HARRIS corner detector proposed by by Harris and Stephens [10], which is an operator to detect corners in images. And the SIFT method (Scale Invariant Feature Transform) proposed by [14] which is a local detector and descriptor. Also, the SURF method (Speed up Robust Features) proposed by [9], is a detector and descriptor that is invariant to change of scale or rotation. It uses integral images, which results in a significant performance boost. However, as we are planning to work with a video file and multiple images in our next work, in this experiment we chose the FAST method to detect the point of interest and used the FLANN method [17] that is available in the OpenCV open source for matching the points of interest that we identified from the detection step. For more detail are shown in below.

1) FAST detector method: A feature from the accelerated segment test (FAST) was originally proposed by [5] [6]. This is a well-known corner detection method. The main idea is to combine edge- and point-based tracking systems to emphasize the problem of a real-time 3D model based on tracking systems. The edge- and point-based systems complement each other and can establish a rather robust system. To carry out a corner detector, a circle of 16 pixels around the corner candidate is considered. The test is performed on a Bresenham circle. The classification of the positive and negative corner is based on the pixels which produce the extreme information and is measured using the ID3 algorithm [21] to determine whether it is a corner. Non-maxima suppression is subsequently applicable on the sum of the absolute difference between the pixels in the circle as well as the center pixel [23].

\section{Suppression of false matching}

The RANSAC algorithm proposed by [7] is intended to verify the matches between key points. The concept of this algorithm is to interpret a method and find its parameters with $N$ subsets of $\mathrm{n}$ random data. From these $N$ estimates of the model, classifying matching points as excellent or weak matches is possible. The pairing validation of points is based on the measurement of error between the projected point from the first image onto the second image as well as the points matched in the second image. The algorithm is used as follows: selecting a set of eight random points and estimating the fundamental matrix, then calculating for each point the distance between the projected point in the second image and the epipolar line. If the distance exceeds a certain threshold, the points are rejected. The remaining items are grouped in $K$ set. The process is iterated $N$ times and the set with the greatest number of elements is selected. Finally, the fundamental matrix with these points is estimated.

\section{E. Delaunay Triangulation}

The Delaunay triangulation is a geometric structure and well know in mesh generation [20]. Given a point set $P$ in the plane, the Delaunay triangulation is a particular triangulation of the points in $P$, which satisfy the empty circum-circle property: the circum-circle of each triangle does not contain any other point $p \in P$. This structure for a set of 3D points is the tetrahedralization of the points in which the circum-sphere of each tetrahedron does not contain any other point of the point set.

Voronoi Diagram (VD) Related to Delaunay triangulation, the Voronoi Diagram (VD) of a set of points is defined as follows: Let $P$ be a set of points in an n-dimensional Euclidean space $R^{n}$. The Voronoi cell of a point $p \in P$, called $V_{p}(P)$, is the set of points $x \in R^{n}$ that are closer to $\mathrm{p}$ than to any other point in $P$ :

$$
V_{p}(P)=x \in R^{n} \mid \quad\|x-p\| \leq\|x-q\|, q \in P, q \neq p
$$

The union of the Voronoi cells of all points $p \in P$ form the Voronoi diagram of $P$, noted as $V D(P)$ :

$$
V D(P)=U V_{p}(P), p \in P
$$

Delaunay triangulation and Voronoi diagrams are dual structures: the center of circum-circles (-spheres) of Delaunay triangulation are the Voronoi vertices; and joining the adjacent generator points in a VD yield their DT. Therefore, having constructed one structure, the other one can be extracted automatically. This duality is very helpful, because construction, manipulation and storage of the VD is more complicated than DT, so all the operations can be operated on DT, and the VD extracted on demand.

\section{F. $3 D$ Reconstruction}

To carry out the $3 \mathrm{D}$ reconstruction, we used all the parameters we have applied previously, including the essential matrix that obtains the pose of the camera from the first image to the second image, the projection matrix $P$ for the first images and $P 2$ for the second ones. Subsequently, the rotation matrix $R$ and the translation vector $t$ are estimated. Finally, a triangulation from the inliers found earlier and the projection matrices is used to create the $3 \mathrm{D}$ reconstruction. The $3 \mathrm{D}$ model is then retained to remove some irregular points that are locally isolated. The 3D Delaunay triangulation was used to create a mesh texture as well as rendering on the 3D model. 


\section{EXPERIMENT AND RESULTS}

In this part some results generated with te proposed system are presented. For the image pair of archeological objects, in this experiment we used a female bust. Firstly, we used the mono camera to take a video of the object in an underwater environment. Secondly, we extracted the file video into an image sequence. Thirdly, as underwater images are normally not very clear, and therefore there is a problem with light, color, and dirty water, we need to improve the quality of the images by using an image processing technique. Fourthly, we choose the image pair for the experiment based on there being enough movement between the image pairs and also enough overlap between them. Finally, the features of interest are detected in each of the images and the points of interest between the image pair are matched. These features are triangulated from the $2 \mathrm{D}$ image into 3D point clouds. The OpenCV open source was used to develop an application to carry out 3D reconstruction, while OpenGL was used for visualization of the 3D model. A 3D industrial scan model was created for the measurement compared with the underwater $3 \mathrm{D}$ reconstruction model.

\section{A. Camera calibration}

In this experiment, the camera used is a Sony CyberShot HD Camera 16.1 megapixel DSC-H70. The resolution used for picture acquisition is $1280 \times 720$ pixels. The camera model in this experiment is a pinhole. Our single camcorder was calibrated by placing a calibration chessboard with know dimensions in the lake floor, and recording a video of it from various angles. Then the camera calibration platform based on [25] and [3] was used. The intrinsic parameters are estimated for each camera, such as the focal length (f), the principal point on $(\mathrm{Cx}, \mathrm{Cy})$, as well as the distortion.

The intrinsic parameters are now known from the calibration, it is used to determine the extrinsic parameters in the Camera Pose Estimation step.

\section{B. Image processing}

The goal of this paper is to work with underwater images. We already know that images taken in an underwater environment always have various noise. As in our original underwater images in this experiment, from the video taken in the lake, there is some effect from white points. So we need to improve our original images to achieve sufficiently good quality to be able to extract and find the points of interest in the images. Initially, we modified the images by gathering the Median filter kernel size 9, in order to reduce the white and black points (salt and pepper points). In addition, we applied a $5 \times 5$ Gaussian kernel to modify blurred images. The Original underwater image and the filtered images are shown in Fig 3.

\section{C. $3 D$ model reconstruction}

The archeological object used in this part to create the 3D model is a female bust. The image pairs used in this experiment were the original images and filtered images. Then the FAST method was used to detect features of interest in the stereo

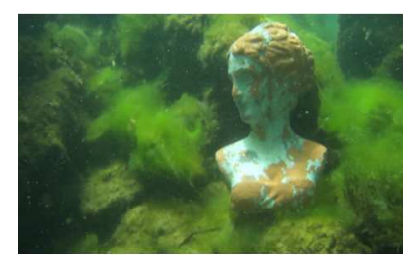

(a) Original Underwater image

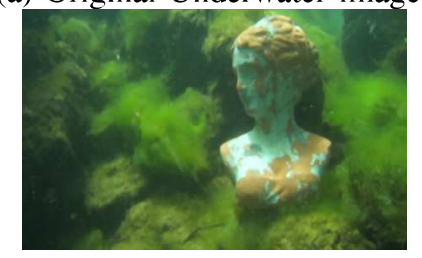

(b) Filtered Underwater image

Fig. 3: (a)The original underwater image and (b) filter underwater image.

images. Subsequently, the FLANN method [18] was used in the matching step to obtain matched points between the first and second images. OpenGL was used for rendering and the textures of the 3D stereo model. As another essential point, the quality of the underwater $3 \mathrm{D}$ reconstruction model was validated by measurements of the reference $3 \mathrm{D}$ scan model and the real object.

1) Detection and Extraction: Robust feature detection and feature matching are crucial to building a robust $3 \mathrm{D}$ model. The matching step is the essential point of the $3 \mathrm{D}$ reconstruction. To begin the detection, the FAST method is used to identify the detected features of interest in the stereo images. The FLANN method is used to match those features in image pairs.Finally, the RANSAC method is used to reject inconsistent matches. Inlier features are points that have a correct match with the initial image pairs. Table I summarizes the results obtained from our experiment.

The results in Table I show that the features of interest detected on the original underwater images are more numerous than in the filtered images. However, not all of the features of interest can be matched between image pairs. And several false matches are represented.Finally, the RANSAC method was used to remove false matched points.

Focused on the percentage of inliers, the results presented in Table I show that in the case of the original underwater images, the percentage of inlier points is $32.93 \%$, which is less than in the case of the filtered underwater images, for which the percentage of inliers is $39.29 \%$. And in the case of the ground image it is $44.47 \%$. From this part of the experiment, we will have $2 \mathrm{D}$ points set to carry out the underwater $3 \mathrm{D}$ reconstruction model in the next step.

From this experiment part, we will have 2D points set to carry out the Underwater $3 \mathrm{D}$ reconstruction model in the next step.

2) Camera Pose Estimation: In the next step, when we have a set of matching points between first and second images. Then we can used them to find the camera movement or we call camera pose. To begining with the fundamental matrix 
TABLE I: Comparison the percent of inlier point.

\begin{tabular}{|c|c|c|c|c|c|}
\hline Object (Bust female) & Image pair & $\begin{array}{c}\text { Number of } \\
\text { features detection }\end{array}$ & $\begin{array}{c}\text { Number of } \\
\text { feature matchinng }\end{array}$ & $\begin{array}{l}\text { Number of } \\
\text { Inliers }\end{array}$ & $\%$ of Inliers \\
\hline \multirow{2}{*}{ Filter underwater image } & Left Image & 2,072 & \multirow{2}{*}{784} & \multirow{2}{*}{308} & \multirow{2}{*}{39.29} \\
\hline & Right Image & 2,390 & & & \\
\hline \multirow{2}{*}{ Original underwater image } & I.eft Image & 7,575 & \multirow{2}{*}{2.317} & \multirow{2}{*}{763} & \multirow{2}{*}{32.93} \\
\hline & Right Image & 8,897 & & & \\
\hline \multirow{2}{*}{ Ground image } & Left Image & 2,826 & \multirow{2}{*}{1,039} & \multirow{2}{*}{462} & \multirow{2}{*}{44.47} \\
\hline & Right Image & 3,504 & & & \\
\hline
\end{tabular}

$F$ is a $3 \times 3$ matrix which relates corresponding points in stereo images. In epipolar geometry, with homogeneous image coordinates, $x$ and $x^{\prime}$, of corresponding points in a stereo image pair, $F x$ describes a line (an epipolar line) on which the corresponding point $x$ on the other image must lie.

$$
x^{\prime T} F x=0
$$

Then the essential matrix method proposed by [11] is used. The aim of this method is to obtain the pose of the second camera with respect to the first camera. The essential matrix $E$ is calculated from fundamental $F$ and calibration $K$ matrices obtained previously.

$$
E=K^{t} x F x K
$$

Calculating the single value decomposition SVD [24] of the essential matrix grants the camera pose $(R$ and $t)$. In the first place is defined the projection matrix $P=K[I \mid 0]$ for the first camera and $P 2=K[R \mid t]$ for the second one.

Subsequently, the rotation matrix $R$ and the translation vector $t$ are estimated.

3) Euclidean Reconstruction: To perform the reconstruction, we use results obtained by the calibration step and the matching points. We used each pair of points and project them in the $3 \mathrm{D}$ space by performing a spacial triangulation.

4) Triangulation and texture: To improve the rendering of the 3D model, texture was added. First, a triangulation of the point cloud is required. We defined here as triangulation, the creation of triangular surface between all the points of the cloud. The Delaunay triangulation is performed from the 2D points. This permit to obtain only visible triangle. Then, they are projected in the $3 \mathrm{D}$ space with the $2 \mathrm{D}$ texture information.

We present the result of the underwater 3D model from the original images and filtered images, turning it in other views to show the model, as seen in Fig 6 and Fig 5. We can see that the 3D model from the filtered images looks smoother than original the image when we turn it around in several views.

\section{Validation with $3 D$ industrial scan model}

The aim of this experiment is to verify the underwater $3 \mathrm{D}$ reconstruction model by comparison with a $3 \mathrm{D}$ industrial scan model that is used as the reference. We used the tool in Meshlab for measurement of our 3D reconstruction model for comparison with the $3 \mathrm{D}$ industrial scan model Fig 7 and the real object. The measurement comparison results are shown in

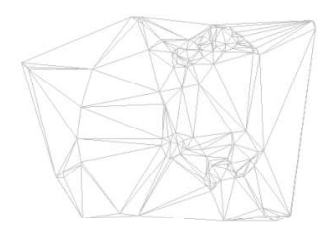

(a) $3 \mathrm{D}$ points

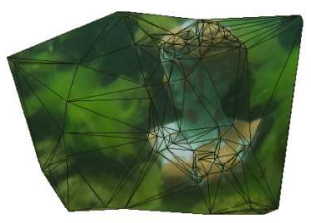

(c) Delaunay with Texture (b) Delaunay from 3D points

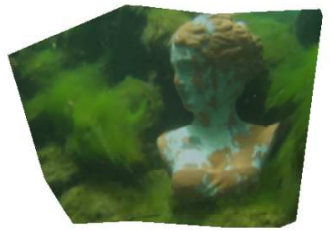

(d) Final 3D model
Fig. 4: The 3D model of filter underwater image.
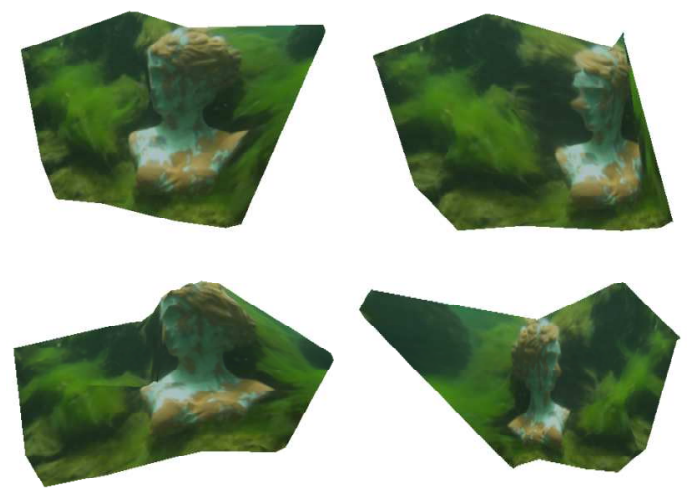

Fig. 5: The final 3D reconstruction model in many views of filtered image.
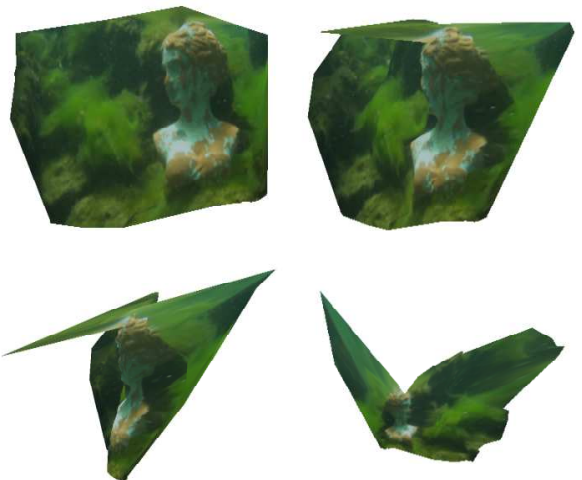

Fig. 6: The final 3D reconstruction model in many views of original image. 
Table II. To summarize, the measurement of the 3D model in the case of filtered underwater images is a robust model, as the measurement result is similar to the real object as well as the $3 \mathrm{D}$ scan model. In part of the nose there is a difference of about $0.23 \mathrm{~cm}$ compared with the 3D scan and about 0.73 $\mathrm{cm}$ compared with the real object. In addition, the top-down (head-bottom) part is different by about $1.8 \mathrm{~cm}$ compared with the $3 \mathrm{D}$ scan and about $1.2 \mathrm{~cm}$ compared with the real object.

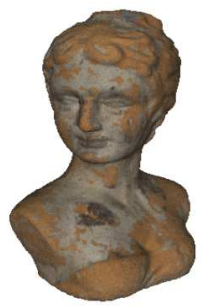

Fig. 7: The 3D scan model.

TABLE II: Table to test captions and labels

\begin{tabular}{|c|c|c|c|c|}
\hline \multirow{2}{*}{ Detail } & \multicolumn{4}{|c|}{ Measurement comparing (unit = cm) } \\
\cline { 2 - 5 } & Real Object & 3D Scan & Original Image & Filtered Image \\
\hline $\begin{array}{c}\text { Top - Down } \\
\text { (head - } \\
\text { bottom) }\end{array}$ & 30 & 29.4 & 38.4 & 31.2 \\
\hline $\begin{array}{c}\text { Left - Right } \\
\text { shoulders }\end{array}$ & 20 & 18.6 & 24.26 & 21.9 \\
\hline Nose & 4 & 3.5 & 4.5 & 3.27 \\
\hline
\end{tabular}

\section{CONCLUSION}

The goal of this work was to achieve a robust $3 \mathrm{D}$ reconstruction of archeological objects with a single camera camcorder system in an underwater environment. To begin with, image processing was used to improve the quality of the underwater image. Then, to achieve this purpose, we have to make sure that the feature points and the matching method are robust enough to the noise condition. Finally, the Euclidian reconstruction method is used to create a 3D model of the archeological object. Finally, the 3D stereo model quality is evaluated by a measurement comparison with a 3D industrial scan model.

In future work, we plan to work with a video of the archeological object obtained with a stereo system instead of a single camera system and to take numerous views of the object to carry out 3D point clouds. Then tracking and 3D mapping techniques will be used to produce a more precise underwater $3 \mathrm{D}$ reconstruction model. Moreover, we will apply the fusion of this $3 \mathrm{D}$ information with a sonar map of an underwater archeological site.

\section{REFERENCES}

[1] Mahiddine Amine, Seinturier Julien, Bo Jean-marc, Drap Pierre, and Merad Djamal. Performances analysis of underwater image preprocessing techniques on the repeatability of sift and surf descriptors. in WSCG 2012: 20th International Conference on Computer Graphics, Visualization and Computer Vision, 2012.

[2] L. Barazzetti. A trip to rome: Physical replicas of historical objects create in a fully automated way from photos. the First int.conf. on Humancomputer interaction, tourism and cultural heritage, HCITOCH'10, (Berlin, Heidelberg), pages 63-72, 2011.
[3] J. Y. Bouguet and P. Perona. Camera calibration from points and lines in dual-space geometry. technical report, California Institute of Technology, 1998.

[4] J. Lim A. Ranganathan M. Pollefeys C. Haene, C. Zach. Stereo depth map fusion for robot navigation. in IEEE/RSJ Int. Conf. on Intelligent Robots and Systems (IROS), 2011.

[5] Reid Porter Edward Rosten and Tom Drummond. Faster and better A machine learning approach to corner detection. IEEE Trans. Pattern Analysis and Machine Intelligence, 32:105-119, 2010.

[6] Tom Drummond Edward Rosten. Machine learning for high-speed corner detection. Computer VisionECCV 2006, pages 430-443, 2006.

[7] M. A. Fischler and R. C. Bolles. Random sample consensus: A paradigm for model fitting with applications to image analysis and automated cartography. Commun. ACM, 24(6):381395, 1981.

[8] M. Goesele, N. Snavely, B. Curless, H. Hoppe, and S. M. Seitz. Multiview stereo for community photo collections. Proceedings of ICCV 2007 , Rio de Janeiro, Brasil, 14-20 October 2007.

[9] T. Tuytelaars H. Bay and L. Van Gool. Surf: Speeded up robust features. In European Conference on Computer Vision, 1(2), May 7-13 2006.

[10] C. Harris and M. Stephens. A combined corner and edge detector. In Proceedings of the Alvey Vision Conference 1998, pages 147-151, 1998.

[11] R. Hartley and A. Zisserman. Multiple view geometry in computer vision. Cambridge University Press, 2003.

[12] A. Hogue, A. German, J. Zacher, and M. Jenkin. Underwater 3d mapping: Experiences and lessons learned. computer and robot vision, Canadian conference, page 24, 07-09 June 2006.

[13] Sahin Isik and Kemal Ozkan. A comparative evaluation of wellknown feature detectors and descriptors. International Journal of Applie Mathematics, Electonics and Computers of computer, 3(1), 2015.

[14] David G. Lowe. Distinctive image feature from scale-invariant keypoints. International Journal of Computer vision, 60(2):91-110, 2004.

[15] A. Meline, J. Triboulet, and B. Jouvencel. A camcorder for 3d underwater reconstruction of archeological objects. OCEANS 2010, 2023 September 2010.

[16] A. Meline, J. Triboulet, and B. Jouvencel. Comparative study of two 3d reconstruction methods for underwater archaeology. Intelligent Robots and Systems (IROS), 2012 IEEE/RSJ International Conference on, pages $740-745,2012$. ISBN: 2153-0858.

[17] Marius Muja and Davi. Scalable nearest neighbor algorithms for high dimensional data. Pattern Analysis and Machine Intelligence (PAMI), 36, 2014.

[18] Marius Muja and David G. Lowe. Fast approximate nearest neighbors with automatic algorithm configuration. International Conference on Computer Vision Theory and Application (VISSAPP'09), pages 331-340, 2009.

[19] Mark S. Nixon and Alberto S. Aguado. Feature extraction and image processing. Academic Press, 2008.

[20] R. Scopigno P. Cignoni, C. Montani. A fast divide and conquer delaunay triangulation algorithm. Computer-Aided Design, 30(5):333341, 1998.

[21] J.R. Quinlan. Induction of decision trees. Maching Learning, pages 81-106, 1986

[22] N. Snavely, Seitz S. M., and R. Szeliski. Photo tourism: exploring photo collections in 3d. ACM Trans. Graph, 25:835-846, July 2006

[23] Tuytelaars T. and K. Mikolajczyk. Local invariant feature detectors: A survey. foundat. Trends Comput. Graphics Vision, pages 177-280, 2008.

[24] Andreas Rechtsteiner Luis M. Rocha Wall, Michael E. Singular value decomposition and principal component analysis. Springer, pages 91109,2003

[25] Zhengyou Zhang. A flexible new technique for camera calibration. IEEE Transactions on Pattern Analysis and Machine Intelligence, 22(11), November 2000 . 